\title{
Estimativa do potencial de perda de fósforo através da metodologia "P Index"
}

\author{
Mariana F. M. de Oliveira', Nerilde Favaretto², Glaucio Roloff² \& Cristóvão V. S. Femandes ${ }^{3}$
}

\begin{abstract}
RESUMO
0 fósforo (P) é considerado o elemento limitante da eutrofização, cuja perda, via escoamento superficial, pode comprometer a qualidade da água. 0 objetivo deste trabalho foi estimar o potencial de perda de $\mathrm{P}$ do solo para a água, por meio da metodologia "P index" (Índice de P - IP) utilizando-se dados experimentais brasileiros e comparar os dados estimados com os valores de P solúvel, P particulado e P total, determinados analiticamente. 0 IP foi desenvolvido nos Estados U nidos da América, em 1993, e tem sido amplamente utilizado em várias versões. Neste artigo se analisaram quatro versões da metodologia IP, utilizando-se dados de publicações científicas desenvolvidas no sul do Brasil, a partir das quais o valor do IP foi obtido e correlacionado com os dados de concentração e perda de P no escoamento superficial. Além da análise de correlação utilizou-se, também, a regressão "stepwise" para avaliar a influência de diferentes componentes do IP na concentração e perda de P. A versão do IP O riginal mostrou as melhores correlações considerando-se a concentração de P no escoamento superficial; já as análises de regressão indicaram que o fator erosão do solo não influenciou na concentração nem na perda de P do solo para a água.
\end{abstract}

Palavras-chave: índice de fósforo, conservação do solo e água, eutrofização

\section{Estimating P loss potencial by P Index}

\begin{abstract}
Phosphorus $(P)$ is the limiting element of eutrophication. The $P$ losses by runoff can affect water quality. The objective of this study was to estimate the P loss from soil to water through the P Index method with Brazilian experimental data and to compare this estimated data with the analytically determined soluble $P$, particulate $P$ and total $P$. The $P$ Index was developed in the U nited States of America, in 1993, and has been widely used in several versions. In this study, four $P$ Index versions were analysed using experiments from southern Brazil. The estimated P Indices were compared with the $P$ concentration and loss in runoff. Besides the correlation analysis, the stepwise regression was used to analyze the influence of different components of the PI in P concentration and loss in the water. The 0 riginal P Index version was more significant with $\mathrm{P}$ concentration in runoff. The regression analyses indicated that the soil erosion factor did not influence P loss from soil to water.
\end{abstract}

Key words. $\mathrm{P}$ index, soil and water conservation, eutrofication

\footnotetext{
1 Engenheira Agrônoma, Mestre em Ciência do Solo. Rua Cap. João Ribas de Oliveira, 415. CEP 81510-350, Curitiba, PR. Fone: (41) $3027-2806$. E-mail: marianafiorin@gmail.com

2 DSEA/UFPR, Rua dos Funcionários 1540, Cabral, CEP 80035-050, Curitiba, PR. Fone: (41) 33505-638. E-mail: nfavaretto@ufpr.br; groloff@ufpr.br

3 DHS/U FPR, Centro Politécnico s/n, Bloco V, Jardim das Américas, CEP 81530-900, Curitiba, PR. E-mail: cris.dhs@ufpr.br
} 


\section{INTRODUÇÃO}

O nitrogênio $(\mathrm{N})$ e o fósforo $(\mathrm{P})$ são os principais nutrientes responsáveis pela eutrofização das águas, mas em função da fixação do $\mathrm{N}$ atmosférico pelas cianobactérias o $\mathrm{P}$ passa a ser o elemento limitante, razão pela qual seu controle é fundamental para proteger e melhorar a qualidade das águas superficiais (Shigaki et al., 2006; Sharpley et al., 2001; Sharpley \& Halvorson, 1994; Sharpley \& Menzel, 1987).

No Brasil, a preocupação com o movimento de nutrientes do solo para a água tem sido enfatizada considerandose a aplicação de dejeto de animais no solo (Mori et al., 2009; Peles, 2007; Shigaki et al., 2006; Bertol, 2005) além de sistemas de preparo e manejo do solo (Bertol et al., 2004b; Leite et al., 2004; Cassol et al., 1999; 2002); no entanto, torna-se necessário, dentre outras demandas, buscar ferramentas de auxílio no planejamento de bacias hidrográficas em relação à poluição das águas por nutrientes, especialmente do $\mathrm{P}$.

O método "P index" (Índice de Fósforo - IP) foi desenvolvido nos Estados Unidos para determinar o risco de perda de P por escoamento superficial e auxiliar no planejamento de bacias hidrográficas. O IP foi proposto, de início, por Lemunyon \& Gilbert (1993) e, desde então, diversas versões deste índice foram desenvolvidas com base nas condições locais e outros fatores de influência no movimento de fósforo do solo para a água (Sharpley et al., 2001). De modo geral, a maioria das versões do IP considera a erosão do solo, o escoamento superficial, o teor de P no solo e o método e a quantidade de $\mathrm{P}$ aplicado, como sendo os principais fatores para a estimativa de perda de $\mathrm{P}$. As principais diferenças entre as versões são: a forma de se calcular o IP e a inclusão de outros fatores que também influenciam na perda de P do solo para a água. Vários estados norte-americanos possuem versões do IP adaptadas às suas condições locais (Sharpley et al., 2003). O IP Original (Lemunyon \& Gilbert, 1993) apresenta uma estrutura de cálculo aditiva visto que os fatores de influência e seus respectivos pesos são somados para chegar ao índice final. Modificações foram feitas no IP original incluindo-se outros fatores e pesos ainda dentro da estrutura de cálculo aditiva (NRCS, 2001; Flynn et al., 2000). Modificações na estrutura de cálculo também foram efetuadas gerando versões multiplicativas, ou seja, para chegar ao índice final se multiplicam os valores dos fatores com seus pesos (Weld et al., 2003).

Em trabalho recente desenvolvido no Brasil, Lopes et al. (2007) utilizaram o IP conduzido por Lemunyon \& Gilbert (1993) com o objetivo de identificar áreas de risco de contaminação de fósforo em bacia hidrográfica porém não existem trabalhos, no País, comparando os índices de P obtidos através da metodologia IP com os dados analiticamente determinados de P no escoamento superficial. Desta forma se propôs, com o presente estudo: 1) estimar o IP obtido de dados contidos em publicações científicas brasileiras, por meio de quatro versões; 2 ) comparar os dados de IP estimado com os de concentração e perda de $\mathrm{P}$ determinados analiticamente e 3) avaliar a influência de diferentes fatores na concentração e perda de $\mathrm{P}$ total, solúvel e particulado do escoamento superficial através de dados de publicações científicas brasileiras.

\section{MATERIAL E MÉTODOS}

Quatro versões do IP foram utilizadas e desenvolvidas nos Estados Unidos da América, em que as escolhidas, foram: o IP Original (Lemunyon \& Gilbert, 1993) (versão aditiva, Tabela 1), a do estado do Alabama (NRCS, 2001) (versão aditiva, Tabela 2), a do Novo México (Flynn et al., 2000) (versão aditiva, Tabela 3) e a da Pensilvânia (Weld et al., 2003) (versão multiplicativa, Tabela 4); sua escolha se deve principalmente por apresentarem informações completas sobre suas estruturas, com fatores e pesos bem definidos, possibilitando a obtenção do IP a partir dos dados experimentais; além disso se observaram as diferentes formas de calcular o IP (aditiva ou multiplicativa); no entanto foi possível, neste trabalho, selecionar somente uma versão multiplicativa; as demais versões multiplicativas existentes não possibilitavam o cálculo com as informações disponíveis nas publicações brasileiras. Na forma aditiva os valores obtidos em cada fator são somados enquanto na forma multiplicativa tais valores são multiplicados entre si e, portanto, a interpretação do valor de IP deve considerar a respectiva versão.

Para comparação dos dados de estimativa de perda de $\mathrm{P}$ obtidos por essas versões de IP buscaram-se, na literatura, todas as publicações científicas brasileiras com dados analíticos de diferentes formas de P na água (concentração e perda de $\mathrm{P}$ nas formas total, solúvel e particulado). Além das determinações analíticas, os trabalhos deveriam conter os seguintes itens para o cálculo do IP: erosão do solo, escoamento superficial, teor de P no solo, taxas e métodos de aplicação de P orgânico e/ou mineral; a partir desses dados se obtiveram os valores de IP pelas diferentes versões possibilitando, assim, a correlação entre os valores de IP obtidos pelas diferentes versões e os resultados de concentração e perda de P. A partir desta seleção foi possível utilizar somente dados de experimentos da região sul do Brasil, contidos nas seguintes publicações: 1) Bertol (2005); 2) Bertol et al. (2004b) e Leite et al. (2004); 3) Badelucci (1997), Cassol et al. (1999) e Cassol et al. (2002); 4) Bertol et al. (2004a) e Guadagnin (2003); 5) Peles (2007) e 6) Bertol et al. (2003), Mello (2002) e Mello et al. (2003).

No experimento 1 (Bertol, 2005), realizado a campo em sistema de plantio direto, sob chuva simulada logo após a aplicação de adubo mineral e orgânico (dejeto líquido de suíno). Os experimentos 2 (Bertol et al., 2004b e Leite et al., 2004) e 4 (Bertol et al., 2004a e Guadagnin, 2003) foram realizados com diferentes métodos de preparo e manejo do solo, sob chuva natural; já o experimento 3 (Badelucci, 1997; Cassol et al., 1999; e, Cassol et al., 2002) foi realizado sob diferentes métodos de preparo do solo, também sob chuva natural, enquanto o experimento 5 (Peles, 2007) foi realizado em laboratório sob chuva simulada logo após a adubação mineral e orgânica (dejeto líquido de suíno). O experimento 6 (Bertol et al., 2003; Mello, 2002 e Mello et al., 2003) foi realizado a campo, sob chuva simulada, com diferentes métodos de manejo do solo. 
Tabela 1. Índice de perda de fósforo - IP O riginal (Lemunyon \& Gilbert, 1993)

\begin{tabular}{|c|c|c|c|c|c|c|}
\hline \multirow{2}{*}{ Fator(1) } & \multirow{2}{*}{ Peso do fator } & \multicolumn{5}{|c|}{ Classificação do fator (valor) } \\
\hline & & Nulo (0) & Baixo (1) & Médio (2) & Alto (4) & Muito alto (8) \\
\hline Teor de P no solo & 1,0 & Nulo ${ }^{(5)}$ & Baixo & Médio & Alto & Excessivo \\
\hline Erosão do solo $\left(\mathrm{t} \mathrm{ha}^{-1}\right)^{(2)}$ & 1,5 & Nula & $<12$ & $12-25$ & $25-37$ & $>37$ \\
\hline Escoamento superficial & 0,5 & Nulo(6) & Muito baixo ou Baixo & Médio & Alto & Muito Alto \\
\hline $\begin{array}{l}\text { Taxa de aplicação de P } \\
\text { mineral }\left(\mathrm{kg} \mathrm{ha}^{-1}\right)^{(3)}\end{array}$ & 0,75 & Não aplicado & $1-34$ & $35-100$ & $101-168$ & $>168$ \\
\hline $\begin{array}{l}\text { Método de aplicação } \\
\text { de P mineral }\end{array}$ & 0,5 & Não aplicado & $\begin{array}{l}\text { No local do plantio com } \\
\text { profundidade maior que } \\
\qquad 5 \mathrm{~cm}^{(4)}\end{array}$ & $\begin{array}{c}\text { Incorporado } \\
\text { imediatamente antes do } \\
\text { plantio }\end{array}$ & $\begin{array}{c}\text { Incorporado }>3 \text { meses } \\
\text { antes do plantio ou } \\
\text { aplicado superficialmente } \\
<3 \text { meses antes do } \\
\text { plantio }\end{array}$ & $\begin{array}{c}\text { Aplicado } \\
\text { superficialmente para } \\
\text { pastagem ou }>3 \text { meses } \\
\text { antes plantio }\end{array}$ \\
\hline $\begin{array}{c}\text { Taxa de aplicação de P } \\
\text { orgânico }\left(\mathrm{kg} \mathrm{ha}^{-1}\right)^{(3)}\end{array}$ & 1,0 & Não aplicado & $1-34$ & $35-67$ & $68-100$ & $>100$ \\
\hline $\begin{array}{l}\text { Método de aplicação de } \\
\text { P orgânico }\end{array}$ & 1,0 & Não aplicado & $\begin{array}{c}\text { Injetado em } \\
\text { profundidade maior que } \\
5 \mathrm{~cm}^{(4)}\end{array}$ & $\begin{array}{c}\text { Incorporado } \\
\text { imediatamente }<3 \\
\text { meses antes do plantio }\end{array}$ & $\begin{array}{c}\text { Incorporado >3 meses } \\
\text { antes do plantio ou } \\
\text { aplicado superficialmente } \\
<3 \text { meses antes do } \\
\text { cultivo }\end{array}$ & $\begin{array}{c}\text { Aplicado } \\
\text { superficialmente em } \\
\text { pastagem ou aplicado } \\
>3 \text { meses antes plantio }\end{array}$ \\
\hline \multicolumn{7}{|c|}{ IP $=\Sigma$ valor do fator $x$ peso do fator } \\
\hline \multicolumn{7}{|c|}{ 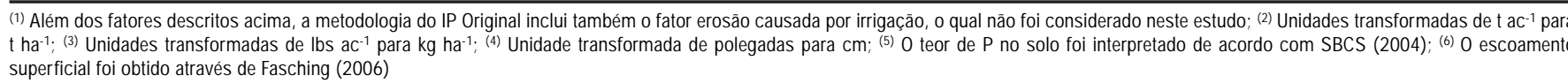 } \\
\hline \multirow[t]{2}{*}{ Fatores $^{(1)}$} & \multirow[t]{2}{*}{ Peso do fator } & \multicolumn{5}{|c|}{ Classificação do fator (valor) } \\
\hline & & Muito baixo (0) & Baixo (1) & Médio (2) & Alto (4) & Muito alto (8) \\
\hline Teor de P no solo & 1,0 & Muito baixo/Baixo(2) & Médio & Alto & Muito Alto & Extremamente Alto \\
\hline $\begin{array}{l}\text { Erosão do solo } \\
\left(\mathrm{t} \mathrm{ha}^{-1} \mathrm{ano}^{-1}\right)^{(3)}\end{array}$ & 3,0 & $<7$ & $7-12$ & $12-25$ & $25-37$ & $>37$ \\
\hline $\begin{array}{l}\text { Taxa de aplicação de P } \\
\left(\mathrm{kg} \mathrm{P}_{2} \mathrm{O}_{5} \mathrm{ha}^{-1} \mathrm{ano}^{-1}\right)^{(4)}\end{array}$ & 3,0 & Não aplicado & $<67$ & $67-135$ & $135-202$ & $>202$ \\
\hline $\begin{array}{c}\text { Método de aplicação } \\
\text { de P }\end{array}$ & 3,0 & Não aplicado & $\begin{array}{l}\text { Injetado em } \\
\text { profundidade maior que } \\
\qquad 5 \mathrm{~cm}^{(5)}\end{array}$ & $\begin{array}{l}\text { Incorporado } \\
\text { imediatamente ou } \\
\text { aplicado com sprinkler }\end{array}$ & $\begin{array}{l}\text { Aplicado } \\
\text { superficialmente e } \\
\text { incorporado }<30 \text { dias }\end{array}$ & $\begin{array}{c}\text { Aplicado } \\
\text { superficialmente, não } \\
\text { incorporado }\end{array}$ \\
\hline $\begin{array}{l}\text { Grupo hidrológico do } \\
\text { solo }\end{array}$ & 3,0 & Nenhum & $A^{(6)}$ & B & C & $\mathrm{D}$ \\
\hline Declividade (\%) & 1,0 & $<1$ & $1-3$ & $3-5$ & $5-8$ & $>8$ \\
\hline
\end{tabular}

(1) Além dos fatores descritos acima, a metodologia considera também os fatores presença de pastagem e de animais, sistemas de drenagem subterrânea, distância de aplicação de $\mathrm{P}$ até 0 rio, largura da vegetação e presença de ambientes críticos ou água degradada, os quais não foram considerados neste estudo; (2) 0 teor de P no solo foi interpretado de acordo com SBCS (2004); (3) Unidades

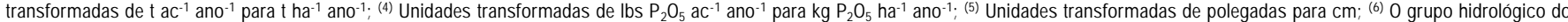
solo foi determinado de acordo com os dados informados na versão do IP Alabama (NRCS, 2001)

Tabela 3. Índice de perda de fósforo - IP N ovo México (Flynn et al., 2000)

\begin{tabular}{|c|c|c|c|c|c|c|}
\hline \multirow{2}{*}{ Fatores ${ }^{(1)}$} & \multirow{2}{*}{ Peso do fator } & \multicolumn{5}{|c|}{ Classificação do fator (valor) } \\
\hline & & Nenhum (0) & Baixo (1) & Médio (2) & Alto (4) & Muito alto (8) \\
\hline $\begin{array}{l}\text { Teor de P no solo } \\
\left(\mathrm{mg} \mathrm{kg}^{-1}\right)\end{array}$ & 1,0 & $\begin{array}{l}\text { Muito Baixo } \\
\quad<8\end{array}$ & $\begin{array}{l}\text { Baixo } \\
8-15\end{array}$ & $\begin{array}{c}\text { Moderado } \\
>15-23\end{array}$ & $\begin{aligned} & \text { Alto } \\
> & 23-30\end{aligned}$ & $\begin{array}{l}\text { Muito Alto } \\
\quad>30\end{array}$ \\
\hline $\begin{array}{l}\text { Erosão do solo } \\
\left(\mathrm{t} \mathrm{ha}^{-1}\right)^{(2)}\end{array}$ & 1,5 & $\begin{array}{l}\text { Muito Baixo } \\
\quad<3\end{array}$ & $\begin{array}{l}\text { Baixo } \\
3-7\end{array}$ & $\begin{array}{l}\text { Médio } \\
>7-12\end{array}$ & $\begin{array}{l}\text { Alto } \\
12-37\end{array}$ & $\begin{array}{l}\text { Muito Alto } \\
>37\end{array}$ \\
\hline Escoamento superficial & 1,5 & Muito Baixo(3) & Baixo & Médio & Alto & Muito Alto \\
\hline $\begin{array}{l}\text { Taxa de aplicação de } \\
\mathrm{P}_{2} \mathrm{O}_{5}\left(\mathrm{~kg} \mathrm{ha}^{-1}\right)^{(4)}\end{array}$ & 1,0 & Não aplicado & $<34$ & $34-100$ & $100-168$ & $>168$ \\
\hline $\begin{array}{l}\text { Método de aplicação de } \\
\text { P mineral }\end{array}$ & 1,0 & Não aplicado & $\begin{array}{l}\text { Local com plantio mais } \\
\text { profundo que } 5 \mathrm{~cm}^{(5)}\end{array}$ & $\begin{array}{l}\text { Incorporado antes do } \\
\text { plantio }\end{array}$ & $\begin{array}{l}\text { Incorporado }>3 \text { meses } \\
\text { antes do plantio ou } \\
\text { aplicado superficialmente } \\
<3 \text { meses antes do } \\
\text { plantio }\end{array}$ & $\begin{array}{c}\text { Aplicado } \\
\text { superficialmente }\end{array}$ \\
\hline $\begin{array}{l}\text { Método de aplicação de } \\
\text { P orgânico }\end{array}$ & 1,0 & Não aplicado & $\begin{array}{l}\text { Injetado de } 8 \text { a } 15 \mathrm{~cm} \\
\text { abaixo da superficiei }\end{array}$ & $\begin{array}{l}\text { Incorporado antes do } \\
\text { plantio }\end{array}$ & $\begin{array}{c}\text { Incorporado }>3 \text { meses } \\
\text { antes do plantio ou } \\
\text { aplicado superficialmente } \\
<3 \text { meses antes do } \\
\text { plantio }\end{array}$ & $\begin{array}{c}\text { Aplicado } \\
\text { superficialmente }\end{array}$ \\
\hline
\end{tabular}

(1) Além dos fatores descritos acima, a metodologia considera também os fatores proximidade do campo ao fluxo de água, erosão por irrigação, manejo de pastagem e área com vegetação/largura, não considerados neste estudo; (2) Unidades transformadas de $\operatorname{tac}^{-1}$ para tha- ${ }^{-1}$; ${ }^{(3)} 0$ escoamento superficial foi interpretado de acordo com Fasching (2006); (4) Unidades transformadas de lbs ac ${ }^{-1}$ para $\mathrm{kg} \mathrm{ha}^{-1}$; (5) Unidade transformada de polegadas para $\mathrm{cm}$ 
Tabela 4. Índice de perda de fósforo - IP Pensilvânia (Weld et al., 2003)

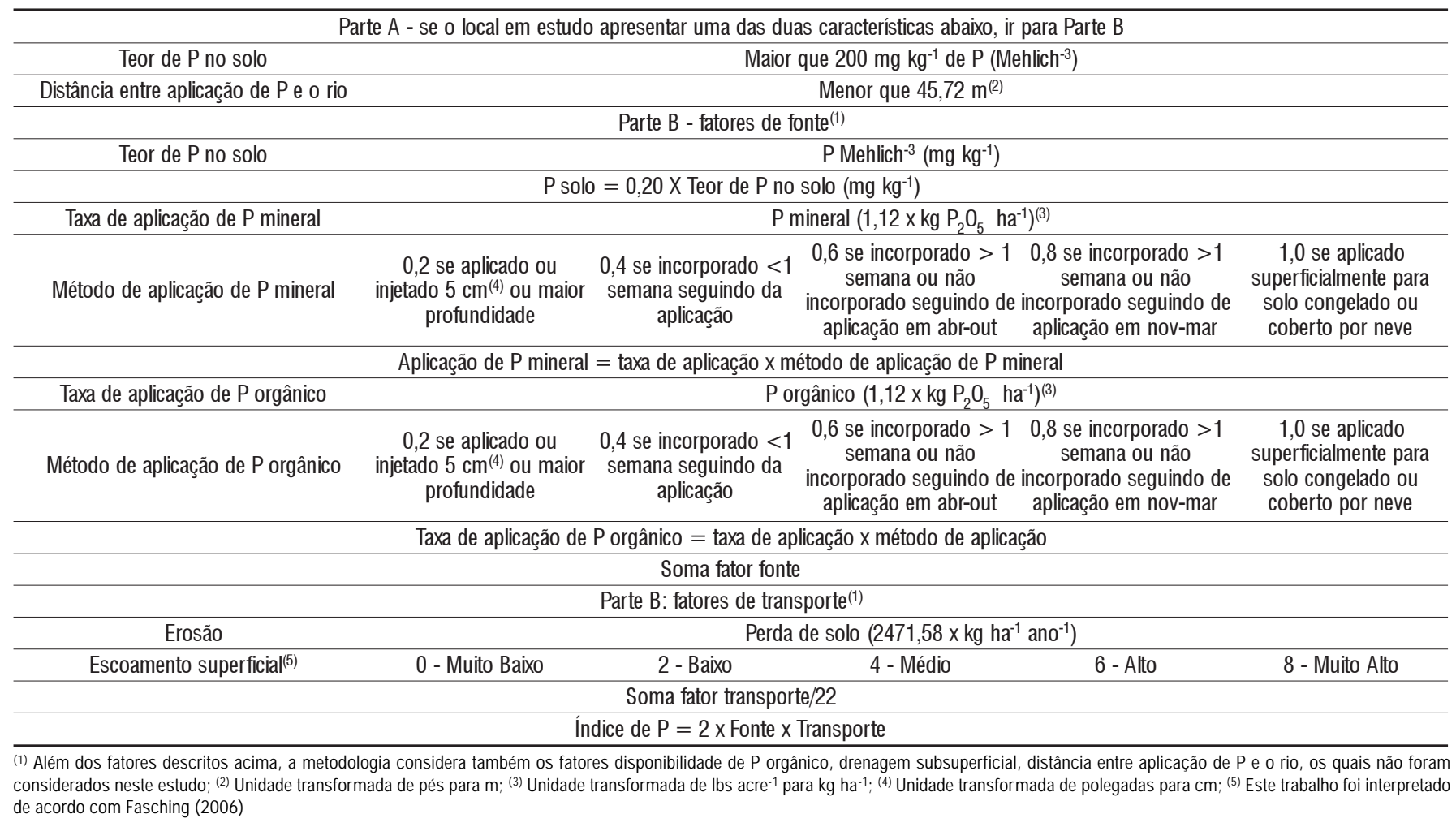

A partir das informações obtidas das publicações acima mencionadas, estimou-se o IP nas quatro versões utilizadas (IP Original, IP Alabama, IP Novo México e IP Pensilvânia. Para estimar o IP, o peso de cada fator foi multiplicado pelo valor do fator e, então, somado ou multiplicado, de acordo com a estrutura de cálculo de cada versão (Tabelas 1, 2, 3 e 4). A interpretação do IP foi feita de acordo com cada versão (Tabela 5); apresenta-se, a seguir, um exemplo de cálculo do IP Original (versão aditiva) para um tratamento do experimento de Bertol (2005):

$\mathrm{IP}=\Sigma$ [(erosão $\mathrm{x}$ peso $)+($ escoamento superficial $\mathrm{x}$ peso $)+($ teor $\mathrm{P}$ solo x peso $)+($ taxa aplicação $\mathrm{P}$ mineral $\mathrm{x}$ peso) + (método aplicação P mineral x peso) + (taxa aplicação P orgânico x peso) + (método aplicação P orgânico $\mathrm{X}$ peso)]

Erosão do solo: $0,126 \mathrm{t} \mathrm{ha}^{-1}$; classificação do fator $=1,0$; peso do fator $=1,5$

Escoamento superficial: classificação do fator = 2,0; peso do fator $=0,5$

Teor de $\mathrm{P}$ no solo: $65,3 \mathrm{mg} \mathrm{kg}^{-1}$; classificação do fator $=8,0$; peso do fator $=1,0$

Taxa de aplicação $\mathrm{P}$ mineral: $0 \mathrm{~kg} \mathrm{ha}^{-1}$; classificação do fator $=0$; peso fator $=0,75$

Método de aplicação P mineral: 0; classificação do fator $=0$; peso do fator $=0,5$

Taxa de aplicação P orgânico: 16,77 kg ha-1; classificação do fator $=2,0$; peso do fator $=1,0$

Método de aplicação $\mathrm{P}$ orgânico: classificação do fator $=8,0$; peso do fator $=1,0$

$\mathrm{IP}=[(1 \mathrm{x} 1,5)+(2 \mathrm{x} 0,5)+(8 \mathrm{x} 1,0)+(0 \mathrm{x} 0,75)+(0 \mathrm{x} 0,5)+$ $(2 \times 1,0)+(8 \times 1,0)]=20,5$
Tabela 5. Interpretação do risco de perda de P para cada versão

\begin{tabular}{|c|c|c|c|c|}
\hline \multirow{2}{*}{ Risco de perda de $P$} & $\begin{array}{c}\mathrm{IP} \\
\text { Original }\end{array}$ & $\begin{array}{c}\mathbb{I P} \\
\text { Alabama }\end{array}$ & $\begin{array}{c}\mathrm{IP} \\
\text { Novo México }\end{array}$ & $\begin{array}{c}\text { IP } \\
\text { Pensilvânia }\end{array}$ \\
\hline & \multicolumn{4}{|c|}{$\begin{array}{l}\text { Valor total (somatório ou multiplicação } \\
\text { dos fatores com o respectivo peso) }\end{array}$} \\
\hline Muito baixo & - & - & $0-10$ & - \\
\hline Baixo & $<8$ & $<65$ & $10-17$ & $0-59$ \\
\hline Médio & $8-14$ & $66-75$ & $17-27$ & $60-79$ \\
\hline Alto & $15-32$ & $76-85$ & $27-37$ & $80-99$ \\
\hline Muito alto & $>32$ & $86-95$ & $37-47$ & $>100$ \\
\hline Excessivo & - & $>96$ & $>47$ & - \\
\hline
\end{tabular}

Realizou-se a análise estatística de Correlação de Pearson utilizando-se o "software SPSS versão 11.0 for Windows”; esta análise de correlação foi feita entre os dados de IP e os dados de concentração e perda de P total, solúvel e particulado do escoamento superficial, obtidos dos trabalhos científicos. $\mathrm{Na}$ análise de correlação se incluíram todos os dados dos experimentos em conjunto, considerando-se as repetições para os tratamentos; realizou-se, posteriormente, a análise de Regressão Múltipla Linear, pela técnica "stepwise” através do "software R" (R Development Core Team, 2006) para verificar o grau de dependência entre a perda de $\mathrm{P}$ por escoamento superficial e os fatores de fonte e transporte de $\mathrm{P}$ do solo para a água. Realizou-se esta análise considerando-se os diferentes fatores do IP (teor de P no solo, erosão do solo, escoamento superficial e taxa e método de aplicação de P mineral e orgânico (Tabela 1), que influenciam na concentração e perda de $\mathrm{P}$ total, solúvel e particulado no escoamento superficial. Da mesma forma que a correlação, a regressão foi realizada considerando-se os dados de 
todos os experimentos. Os fatores considerados nesta análise de regressão foram os seguintes: $\mathrm{a}=$ Teor de $\mathrm{P}$ no solo $\left(\mathrm{mg} \mathrm{kg}{ }^{-1}\right) ; \mathrm{b}=$ Taxa de aplicação de $\mathrm{P}$ mineral $\left(\mathrm{kg} \mathrm{ha}^{-1}\right)$; $\mathrm{c}=$ Método de aplicação de $\mathrm{P}$ mineral $(0=$ não aplicado, 1 = aplicado incorporado, 2 = aplicado superficialmente); d = Taxa de aplicação de P orgânico $\left(\mathrm{kg} \mathrm{ha}^{-1}\right)$; e = Método de aplicação de $\mathrm{P}$ orgânico ( 0 = não aplicado, 1 = aplicado incorporado, 2 = aplicado superficialmente); $\mathrm{f}$ = Erosão do solo $\left(\mathrm{kg} \mathrm{ha}^{-1}\right)$; e g = Escoamento superficial (mm).

\section{RESULTADOS E DISCUSSÃO}

O IP Original foi o que apresentou as melhores correlações para as concentrações de P solúvel, total e particulado no escoamento superficial (Tabela 6); as demais versões não indicaram correlações significativas, com exceção do IP Novo México. Apesar do teste de correlação ser, de modo geral, significativo, os coeficientes de correlação foram muito baixos, variando de 0,31 a 0,48 (Tabela 6). As baixas correlações são explicadas pela grande variabilidade dos dados analisados os quais envolveram experimentos com chuva natural e simulada, adubação mineral e orgânica bem como métodos de preparo e manejo do solo (Eghball \& Gilley, 2001).

Tabela 6. Índice de correlação de Pearson ( $r$ ) entre P solúvel (PS), particulado (Pp) e total (Pt) e os valores de IP Alabama (IP A), IP Novo México (IP N M), IP O riginal (IP O) e IP Pensilvânia (IP P)(1)

\begin{tabular}{|c|c|c|c|c|c|c|}
\hline \multirow{3}{*}{ Índice IP } & $\mathrm{Ps}^{(1)}$ & $\mathrm{Pp}$ & $\mathrm{Pt}$ & Ps & $\mathrm{Pp}$ & Pt \\
\hline & & $\mathrm{mg} \mathrm{L}^{-1}$ & & \multicolumn{3}{|c|}{$\mathrm{g} \mathrm{ha}^{-1}$} \\
\hline & \multicolumn{6}{|c|}{$r$} \\
\hline IP A & 0,24 & $-0,05$ & 0,17 & 0,05 & 0,15 & 0,12 \\
\hline IP NM & $0,31^{*}$ & 0,31 & 0,19 & 0,12 & 0,33 & $0,33^{*}$ \\
\hline IP 0 & $0,46^{*}$ & $0,48^{*}$ & $0,45^{\star}$ & 0,25 & 0,42 & 0,23 \\
\hline IP P & 0,07 & $-0,15$ & $-0,08$ & $-0,04$ & 0,13 & 0,22 \\
\hline
\end{tabular}

* significativo para $\mathrm{p}$-valor $<0,05$

(1) Para a correlação utilizou-se a média dos tratamentos sendo que para Ps (mg L-1), Pp (mg L-1), $\mathrm{Pt}\left(\mathrm{mg} \mathrm{L}^{-1}\right)$, Ps ( $\left.\mathrm{g} \mathrm{ha}^{-1}\right), \mathrm{Pp}\left(\mathrm{g} \mathrm{ha} \mathrm{-}^{-1}\right)$ e Pt ( $\left.\mathrm{g} \mathrm{ha} \mathrm{a}^{-1}\right)$ as médias envolvidas foram $56,21,53,36,21 \mathrm{e}$ 73 , respectivamente

Os fatores do IP Original que possuem maior peso são a erosão do solo, o teor de $\mathrm{P}$ no solo e a taxa e o método de aplicação de P orgânico (Tabela 1); esses fatores, com exceção da erosão do solo, foram os mesmos indicados nas análises de regressão como os de maior influência no movimento de $\mathrm{P}$ do solo para a água (Tabela 7); pode-se considerar, portanto, que o IP Original apresentou resultados que mais se aproximaram da condição desses experimentos analisados conjuntamente.

O teor de $\mathrm{P}$ no solo, o método de aplicação de $\mathrm{P}$ mineral, a taxa e o método de aplicação de P orgânico e o escoamento superficial, são os fatores que explicam a concentração e perda de P solúvel (Tabela 7); pode-se considerar, portanto, que a versão do IP que indicaria maior proximidade da perda real de $\mathrm{P}$, seria aquela em que os pesos para tais fatores fossem maiores.

Nos resultados de $\mathrm{P}$ particulado, cujos dados se referem
Tabela 7. Regressão "stepwise" de todos os experimentos indicando os efeitos das variáveis selecionadas, tanto em concentração ( $\left.\mathrm{mg} \mathrm{L}^{-1}\right)$ como em perda ( $\mathrm{g}$ ha-1) de $\mathrm{P}$ solúvel (Ps), particulado (Pp) e total (Pt)

\begin{tabular}{|c|c|c|c|c|c|c|}
\hline \multirow{3}{*}{ Variável } & $\mathrm{Ps}^{(1)}$ & $\mathrm{Pp}$ & Pt & Ps & $\mathrm{Pp}$ & $\mathrm{Pt}$ \\
\hline & \multicolumn{3}{|c|}{$\mathrm{mg} \mathrm{L}^{-1}$} & \multicolumn{3}{|c|}{$\mathrm{g} \mathrm{ha}^{-1}$} \\
\hline & \multicolumn{6}{|c|}{$R^{2}$ parcial } \\
\hline Teor P no solo & 0,23 & 0,50 & 0,22 & 0,05 & 0,04 & 0,00 \\
\hline $\begin{array}{l}\text { Método de } \\
\text { aplicação de P } \\
\text { mineral }\end{array}$ & 0,09 & - & 0,01 & 0,06 & - & 0,02 \\
\hline $\begin{array}{c}\text { Taxa de aplicação } \\
\text { de P orgânico }\end{array}$ & 0,06 & - & - & 0,18 & 0,33 & - \\
\hline $\begin{array}{c}\text { Método de } \\
\text { aplicação de P } \\
\text { orgânico }\end{array}$ & 0,04 & 0,19 & 0,21 & 0,05 & - & 0,09 \\
\hline $\begin{array}{l}\text { Escoamento } \\
\text { superficial }\end{array}$ & 0,07 & - & - & 0,02 & 0,30 & - \\
\hline $\begin{array}{c}\text { Taxa de aplicação } \\
\text { de P mineral }\end{array}$ & - & - & 0,15 & 0,01 & - & 0,17 \\
\hline Total & 0,49 & 0,69 & 0,58 & 0,37 & 0,67 & 0,28 \\
\hline
\end{tabular}

(1) Para a correlação utilizou-se a média dos tratamentos sendo que para $\mathrm{Ps}\left(\mathrm{mg} \mathrm{L}^{-1}\right), \mathrm{Pp}\left(\mathrm{mg} \mathrm{L}^{-1}\right)$, $\mathrm{Pt}\left(\mathrm{mg} \mathrm{L}^{-1}\right), \mathrm{Ps}\left(\mathrm{g} \mathrm{ha}^{-1}\right), \mathrm{Pp}\left(\mathrm{g} \mathrm{ha} \mathrm{a}^{-1}\right)$ e Pt ( $\left.\mathrm{g} \mathrm{ha} \mathrm{a}^{-1}\right)$ as médias envolvidas foram $56,21,53,36,21 \mathrm{e}$ 73 , respectivamente

apenas aos experimentos com adubação orgânica, nota-se diferença entre concentração $\left(\mathrm{mg} \mathrm{L}^{-1}\right)$ e perda de $\mathrm{P}\left(\mathrm{g} \mathrm{ha}^{-1}\right)$. Observa-se que o teor de $\mathrm{P}$ no solo e o método de aplicação de P orgânico são os principais fatores que influenciam na concentração de P particulado no escoamento superficial enquanto a taxa de aplicação de $\mathrm{P}$ orgânico e o escoamento superficial explicam 63\% da perda de P particulado (Tabela 7). Desta forma se considera que, para trabalhos nos quais são utilizadas adubações orgânicas com chuva imediatamente após a adição do dejeto, se deve escolher um IP com pesos maiores para os fatores taxa e método de aplicação de P orgânico; já no estudo de Eghball \& Gilley (2001), as taxas de aplicação de P orgânico não influenciaram significativamente na perda de $\mathrm{P}$ particulado, apesar das taxas de aplicação terem sido maiores que as recomendadas para a área.

Diferentemente do estudo de Eghball \& Gilley (2001), cujo teor de $\mathrm{P}$ no solo contribuiu com apenas $1 \%$ da perda de $\mathrm{P}$ total, no presente estudo o teor de $\mathrm{P}$ no solo foi um dos principais fatores que contribuíram tanto para a perda quanto para a concentração de $\mathrm{P}$ ( $\mathrm{g} \mathrm{ha}^{-1}$ e $\mathrm{mg} \mathrm{L}^{-1}$ ) (Tabela 7). Além deste fator, a taxa de aplicação de $\mathrm{P}$ mineral e o método de aplicação de $\mathrm{P}$ mineral e de $\mathrm{P}$ orgânico também influenciaram na perda de $\mathrm{P}$ total; portanto, quando se está considerando a perda de $\mathrm{P}$ total pode-se utilizar um IP no qual esses fatores possuam maiores pesos.

O teor de P no solo contribuiu com 23, 50 e 22\% da concentração de $\mathrm{P}$ solúvel, particulado e total, respectivamente (Tabela 7). O fator método de aplicação de P mineral afetou, de forma mínima (menos que 9\%) na concentração e perda de $\mathrm{P}$ do solo para a água. O fator método de aplicação de P orgânico contribuiu com 19 e 21\% na concentração de $\mathrm{P}$ particulado e total, respectivamente. A taxa de aplicação de P orgânico contribuiu com 18 e 33\% na perda de P solúvel e particulado, respectivamente.

No estudo de Eghball \& Gilley (2001), a erosão do solo foi o fator que mais contribuiu para as perdas de $\mathrm{P}$ total e 
particulado, ao contrário do observado neste estudo, em que referido fator não apresentou influência significativa nas perdas de $\mathrm{P}$ devido, possivelmente, aos baixos valores de perda de solo na maioria dos tratamentos utilizados.

Sugere-se, a partir desses resultados, a continuidade desta pesquisa, através de experimentos aplicados em campo e se utilizando as características brasileiras e os fatores do IP, para sua posterior aplicação; além disso, torna-se necessário desenvolver um IP brasileiro para que seja possível estimar a perda de $\mathrm{P}$ do solo para a água, de forma mais precisa e significativa, de acordo com as características específicas existentes no sul do Brasil.

\section{CONCLUSÕES}

1. A versão do IP Original apresentou as melhores correlações, apesar dos baixos valores, indicando ser esta a melhor versão para estimar o potencial de perda de $\mathrm{P}$.

2. O teor de P no solo e o método de aplicação de P orgânico foram os principais fatores que influenciaram na concentração de P no escoamento superficial.

3. A quantidade perdida de $\mathrm{P}$ via escoamento superficial foi influenciada pela taxa de aplicação de P orgânico e mineral e o escoamento superficial.

\section{LITERATURA CITADA}

Badelucci, M. P. Infiltração de água e perdas por erosão em diferentes métodos de melhoramento da pastagem nativa. Porto Alegre: UFRGS. 1997, 140p. Dissertação Mestrado

Bertol, I.; Guadagnin, J. C.; Cassol, P. C.; Amaral, A. J.; Barbosa, F. T. Perdas de fósforo e potássio por erosão hídrica em um Inceptisol sob chuva natural. Revista Brasileira de Ciência do Solo, v.28, p.485-494, 2004a.

Bertol, I.; Leite, D.; Guadagnin, J. C.; Ritter, S. R. Erosão hídrica em um Nitossolo Háplico submetido a diferentes sistemas de manejo sob chuva simulada. II. Perdas de nutrientes e carbono orgânico. Revista Brasileira de Ciência do Solo, v.28, p.1045-1054, 2004b.

Bertol, I.; Mello, E. L.; Guadagnin, J. C.; Zaparolli, A. L. V.; Carrafa, M. R. Nutrient losses by water erosion. Scientia Agricola, v.60, p.581-586, 2003.

Bertol, O. Contaminação da água de escoamento superficial e da água percolada pelo efeito de adubação mineral e adubação orgânica em sistema de semeadura direta. Curitiba: UFPR, 2005. 209p. Tese Doutorado

Cassol, E. A.; Levien, R.; Anghinoni, L.; Badelucci, M. P. Perdas de nutrientes por erosão em diferentes métodos de melhoramento de pastagem nativa no Rio Grande do Sul. Revista Brasileira de Ciência do Solo, v.26, p.705-712, 2002.

Cassol, E. A.; Levien, R.; Jong Van Lier, Q.; Badelucci, M. P. Infiltração de água e perdas de água e solo por erosão influenciadas por diferentes métodos de melhoramento da pastagem nativa gaúcha. Revista Brasileira de Ciência do Solo, v.23, n.4, p.923-931, 1999.
Eghball, B.; Gilley, J. E. Phosphorus risk assessment index evaluation using runoff measurements. Journal of Soil and Water Conservation, v.56, n.3, p.202-206, 2001.

Fasching, R. A. Phosphorus index assessment for Montana. Ecological Sciences - Agronomy Technical Note. Montana: Natural Resources Conservation Service (NRCS). Nutrient Management.2006. 7p. Agronomy Technical Note MT-77 (Rev.3).

Flynn, R.; Sporcic, M.; Scheffe, L. Phosphorus assessment tool for New Mexico. U.S. Albuquerque: Department of Agriculture-Natural Resources Conservation Service (NRCS). Publications Distribution Center, NRCS, 2000. 4p. Technical Note Agronomy-57

Guadagnin, J. C. Perdas de nutrientes e carbono orgânico pela erosão hídrica, em um Cambissolo Húmico alumínico léptico submetido a diferentes sistemas de preparo e cultivo do solo. Lages: UDESC, 142p. 2003. Dissertação Mestrado

Leite, D.; Bertol, I.; Guadagnin, J. C.; Santos, E. J.; Ritter, S. R. Erosão hídrica em um Nitossolo Háplico submetido a diferentes sistemas de manejo sob chuva simulada. I. Perdas de solo e água. Revista Brasileira de Ciência do Solo, v.28, p.1033-1044, 2004.

Lemunyon, J. L.; Gilbert, R. G. The concept and need for a phosphorus assessment tool. Journal of Production Agriculture, v.6, p.483-486, 1993.

Lopes, F.; Merten, G. H.; Franzen, M.; Giasson, E.; Helfer, F.; Cybis, L. F. A. Utilização de P-Index em uma bacia hidrográfica através de técnicas de geoprocessamento. Revista Brasileira de Engenharia Agrícola e Ambiental, v.11, n.3, p.312-317, 2007.

Mello, E. L. Erosão hídrica em diferentes sistemas de manejo do solo sob chuva simulada. Lages: UDESC, 2002. 81p. Dissertação Mestrado

Mello, E. L.; Bertol, I.; Zaparolli, A. L. V.; Carrafa, M. R. Perdas de solo e água em diferentes sistemas de manejo de um Nitossolo Háplico submetido à chuva simulada. Revista Brasileira de Ciência do Solo, v.27, p.901-909, 2003.

Mori, H. F.; Favaretto, N.; Pauletti, V.; Dieckow, J.; Santos, W. L. Perda de água, solo e fósforo com aplicação de dejeto líquido bovino em Latossolo sob plantio direto e com chuva simulada. Revista Brasileira de Ciência do Solo, v.33, p.189-198, 2009.

NRCS - Natural Resources Conservation Service. Phosphorus index for Alabama: A planning tool to assess and manage $\mathrm{P}$ movement. Auburn: U.S. Department of Agriculture - Natural Resources Conservation Service. 2001. 5p. Agronomy Technical Note AL-72

Peles, D. Perdas de solo, água e nutrientes sob aplicação de gesso e dejeto líquido de suínos. Curitiba, UFPR, 2007. 97p. Dissertação Mestrado

R Development Core Team. R: A language and environment for statistical Computing. Vienna, 2006. R Foundation for Statistical Computing.

SBCS - Sociedade Brasileira de Ciência do Solo. Manual de adubação e de calagem para os Estados do Rio Grande do Sul e de Santa Catarina / Sociedade Brasileira de Ciência do Solo. 10.ed, Porto Alegre: SBCS, 2004. 400p. 
Sharpley, A. N.; Halvorson, A. D. The management of soil phosphorus availability and its impacto on surface water quality. In: Lal, R; Stewart, B. A. (ed.). Soil processes and water quality. Boca Raton: Lewis Publishers, 1994. p.1-84.

Sharpley, A. N.; Mcdowell, R. W.; Kleinman, P. J. A. Phosphorus loss from land to water: integrating agricultural and environmental management. Plant and Soil, v.237, p.287-307, 2001.

Sharpley, A. N.; Menzel, R. G. The impact of soil and fertilizer phosphorus on the environment. Advances in Agronomy, v.41, p.297-324, 1987.
Sharpley, A. N.; Weld, J. L.; Beegle, D. B.; Kleinman, P. J. A.; Gburek, W. J.; Moore Jr, P. A.; Mullins, G. Development of phosphorus indices for nutrient management planning strategies in the United States. Journal of Soil and Water Conservation, v.58, n.3, p.137, 2003.

Shigaki, F.; Sharpley, A.; Prochnow, L. I. Animal-based agriculture, phosphorus management and water quality in Brazil: Options for the future. Scientia Agricola, v.63, n.2, p.194-209, 2006.

Weld, J. L.; Beegle, D. B.; Gburek, W. L.; Kleinman, P. J. A.; Sharpley, A. N. The Pennsylvania phosphorus index: Version 1. Pennsylvania: Publications Distribution Center, Pennsylvania State University, University Park, 2003. 24p. 